\title{
Predictores de dominio específico para la fluidez de cálculo al inicio de la Educación Primaria
}

\section{Estíbaliz Aragón $^{1}$, José I. Navarro ${ }^{2}$, Manuel Aguilar ${ }^{3}$}

123 Departamento de Psicología, Universidad de Cádiz, Puerto Real

España

Correspondencia: Estibaliz Aragón; Departamento de Psicología. Universidad de Cádiz. Campus Río San Pedro; 11519 Puerto Real-Cádiz (España); estivaliz.aragon@uca.es

Agradecimientos: Este trabajo fue parcialmente financiado por el proyecto I+D+i PSI2015-63856-P (MINECO/FEDER).

(C) Education \& Psychology I+D+i and Ilustre Colegio de la Psicología de Andalucía Oriental (Spain) 


\section{Resumen}

Introducción. El presente estudio analizó el papel predictor sobre la fluidez de cálculo de distintos predictores específicos que conforman las habilidades numéricas tempranas.

Método. Un total de 122 alumnos $(M=69.89$ meses; DT = 3.44), de los cuales 54 fueron niñas y 68 niños. Las habilidades numéricas fueron evaluadas al finalizar la educación infantil y la fluidez de cálculo en el primer curso de educación primaria.

Resultados. El resultado del análisis de regresión lineal arrojó un modelo predictivo que explicaba el $27.9 \%$ de la varianza de la fluidez de cálculo. El conteo verbal $(\beta=.377)$, conteo resultante $(\beta=.191)$ y estimación $(\beta=.159)$, fueron la variables que explicaron en mayor medida la fluidez de cálculo en $1^{\circ}$ de Educación Primaria.

Discusión y Conclusión. Las habilidades numéricas predijeron la fluidez de cálculo, sin embargo, no fue así en el caso de las habilidades relacionales. Se discute la importancia del conteo como dominio para el aprendizaje de las matemáticas.

Palabras Clave: Fluidez de cálculo, Habilidades numéricas tempranas, Predictores específicos, Conteo. 


\section{Abstract}

Introduction. This study analyses the predictive role of several specific components of early numerical skills on the fluency calculation.

Method. A total of 122 students (Mean age $=69.89$ months; SD = 3.44), 54 girls and 68 boys participated in the study. The early numeracy skills were assessed by the end of pre primary school education, and fluency calculation at the first year of primary school.

Results. A multiple linear stepwise regression analysis showed a predictive model that explained $27.9 \%$ of the variance in fluency calculation. This model suggested that verbal counting $(\beta=.377)$, resulting counting $(\beta=.191)$ and estimation $(\beta=.159)$, were the most important numerical variables explained fluency calculation in first graders primary school.

Discussion y Conclusiones. Numerical skills predicted fluency calculation, however, was not predicted by relational math skills. The extensive importance of fluency calculation on learning mathematics is discussed.

Keywords: Fluency calculation, Early numeracy, Domain-specific predictors, Counting 


\section{Introducción}

Obtener un buen rendimiento matemático es uno de los objetivos más importantes para el alumnado de la educación primaria. Presentar dificultades en el aprendizaje de las matemáticas puede tener consecuencias para la vida cotidiana y el futuro escolar de un niño. Realizar una identificación temprana de los niños en riesgo de dificultades permite tratarlos tempranamente (Aragón, Aguilar, Navarro, y Araújo, 2015; Núñez del Río y Lozano Guerra, 2003), y esto puede tener consecuencias positivas para su vida años después (Clements y Sarama, 2011). El pensamiento matemático, se considera como una parte esencial dentro de las funciones cognitivas humanas. Algunas investigaciones se han centrado en el estudio de las diferencias individuales y la identificación temprana de los alumnos en riesgo (Aragón, Delgado, Aguilar, Araujo y Navarro, 2013; Navarro, Aguilar, García, Menacho, Marchena y Alcalde, 2010). Actualmente, estas diferencias y el rendimiento en las destrezas matemáticas se deberían a dos tipos de factores predictores. Passolunghi, Lanfranchi, Altoè, y Sollazzo (2015) consideran, por un lado, la importancia de los predictores de dominio general, que consisten aquellos procesos cognitivos superiores que predicen el rendimiento en materias escolares y no en un solo dominio de contenido concreto: ejemplos de este tipo de elementos cognitivos son la inteligencia general y la memoria de trabajo (Bull, Espy y Wiebe, 2008; Aragón, Navarro, Aguilar y Cerda, 2015). Por otro lado, los predictores de dominio específico se entienden como aquellas habilidades que predicen el desempeño en un área en concreto, por ejemplo las habilidades de conteo en matemáticas (De Smedt, Janssen, Bouwens, Verschaffel, Boets y Ghesquière, 2009).

Esta clasificación entre factores generales y específicos es fundamental para llegar a explicar el hecho de que determinados niños tengan importantes dificultades en matemáticas a pesar de manifestar un óptimo funcionamiento en los predictores de dominio general (Landerl, Bevan, y Butterworth, 2004). No cabe duda sobre la importancia de los predictores específicos en el éxito en matemáticas y especialmente en el cálculo. Por ejemplo, podemos mencionar que tradicionalmente, la habilidad de los niños para recitar la secuencia numérica se alza como un fuerte predictor del rendimiento en tareas numéricas, tales como la resolución de sumas simples (Martins-Mourao y Cowan, 1998; Ho y Fuson, 1998), resultados que se mantienen en la investigación más reciente (Cowan y Powell, 2014; Johansson 2005). 
Las destrezas que predicen el rendimiento de los niños en matemáticas se engloban bajo el concepto denominado habilidades numéricas tempranas. Dichas habilidades comprenderían destrezas tanto de tipo relacional, por ejemplo realizar las comparaciones; como numéricas propiamente dichas, tales como el conteo y la estimación en una línea numérica mental (Gersten, Jordan y Flojo, 2015). Existen numerosas investigaciones que apoyan la relación entre las habilidades numéricas tempranas y el desempeño del alumnado en cursos posteriores (Clements y Sarama, 2009; Sarama y Clements, 2009). Particularmente, en relación al papel predictivo de las habilidades numéricas tempranas, en el correcto desempeño del cálculo en los inicios de la educación primaria (Jordan, Kaplan, Locuniak y Ramineni 2007; Locuniak y Jordan, 2008) y durante el desarrollo de la misma (Cowan y Powell, 2014; Koponen, Aunola, Ahonen y Nurmi 2007; Mazzocco y Thompson 2005).

Llegados a este punto cabe mencionar que, aunque un cálculo correcto y preciso es un objetivo a alcanzar por el alumnado, y un propósito fundamental para los profesores, también se persigue como meta alcanzar la fluidez en el mismo. Podemos entender como fluidez a aquella facilidad y precisión con la que nos desenvolvemos en una tarea determinada. En nuestro ámbito, la fluidez en el cálculo se referiría a la destreza a la hora de llevar a cabo una operación aritmética, de manera flexible, precisa, eficiente y apropiada (Kilpatrick, Swafford y Findell 2001), siendo éstas características clave en numerosas situaciones cotidianas y escolares, tales como la resolución de problemas aritméticos (Fuchs et al., 2006, 2010). Por tanto, alcanzar un nivel óptimo de fluidez en las operaciones básicas, abre camino al alumnado para llevar a cabo tareas más complejas, como las operaciones multidígitos, siendo esencial el dominio de las combinaciones básicas de números pequeños y aquellas combinaciones complejas de dos o más números.

Asimismo, se debe tener presente que las combinaciones básicas van a requerir un conocimiento específico de las relaciones entre los números basadas en reglas, conceptos y principios, tales como los principios del conteo y la reversibilidad (Gelman y Gallistel, 1978). De este modo, aquellos alumnos que tras el primer año de primaria, no alcanzan el dominio de las combinaciones aditivas más básicas, tendrán importantes dificultades en la adquisición y fluidez a la hora de realizar sustracción, multiplicación y división. En consecuencia, su desempeño en cualquier tipo de requerimiento de tipo aritmético, tanto mental como escrito, se verá sensiblemente afectado en comparación con sus iguales (National Mathematics Advisory Pa- 
nel, 2008) y traerá consigo inconvenientes para la instrucción en nociones matemáticas de mayor complejidad y en el desarrollo del razonamiento aritmético superior (Gersten et al., 2005).

El éxito en la resolución de combinaciones numéricas básicas requiere de una serie de reglas y conceptos que no pueden reducirse exclusivamente a la mera memorización de hechos numéricos. Acorde con la investigación sobre el sentido numérico, la memorización de las combinaciones básicas implicaría para un funcionamiento fluido y óptimo la construcción de un cuerpo de conocimiento bien estructurado e interconectado con aquellas concepciones matemáticas elementales (Baroody, Bajwa, y Eiland, 2009). Estos mismos autores inciden en que la principal causa de problemas con las combinaciones básicas y, en consecuencia, de la fluidez de cálculo, se encuentra estrechamente relacionada con el desarrollo del sentido numérico, y las habilidades que lo constituyen en los años previos a la educación formal.

Es por ello que la investigación no debe centrarse exclusivamente en aquellos precursores de dominio general, como por ejemplo la memoria (Koponen et al., 2007), sino prestar atención al mismo tiempo a la importancia de los precursores de dominio específico, y su correspondiente peso explicativo en la fluidez de cálculo, ya que contribuyen de manera independiente a la explicación de la varianza en la fluidez de cálculo en primaria (Cowan et al., 2014). En definitiva, el estudio pormenorizado de los componentes específicos que constituyen las habilidades numéricas tempranas y su papel predictor en la fluidez de cálculo, constituye el objetivo principal de la presente investigación.

\section{Objetivos e hipótesis}

El objetivo general de esta investigación ha sido analizar si los predictores específicos del aprendizaje matemático evaluados al final del ciclo de Educación Infantil (5 años) están relacionados con la fluidez de cálculo a los 6 años de edad. La hipótesis planteada fue la existencia de una relación predictiva entre los precursores específicos del aprendizaje matemático y la fluidez de cálculo al final del primer curso de Educación Primaria. 


\section{Método}

\section{Participantes}

La muestra de estudiantes pertenecía a cuatro centros escolares, dos de carácter concertado y dos públicos. Los centros se situaban en una zona con nivel socio-económico medio. Los participantes fueron un total de 122 alumnos/as pertenecientes al último curso de Educación Infantil en la primera toma de datos, y de primer curso de Educación Primaria en la segunda etapa de recogida de datos.

Las edades de los alumnos en la evaluación mediante el TEMT-i oscilaban entre los 64 y los 76 meses, con una media de 69.89 meses y una desviación típica de 3.44. Del total de la muestra, 54 participantes fueron niñas, cuyas edades oscilaron entre los 64 y 76 meses $(M=$ 69.94; $d t=3.58$ ). Los participantes varones fueron 68 cuyas edades oscilaron entre 64 y 76 meses $(M=69.84 ; d t=3.58)$. La segunda evaluación se llevó a cabo con la misma muestra 12 meses después.

\section{Instrumentos}

Early Numeracy Test (Test de Evalaución Matemática Temprana-informatizado: TEMT-i). Se empleó una prueba destinada a la evaluación del conocimiento matemático temprana a alumnado de 4 a 7 años en su versión española computerizada (Van Luit et al., 2015), la cual supera las limitaciones de la versión lápiz y papel y añade ventajas derivada del uso de las nuevas tecnologías en la evaluación matemática temprana (Araújo, Aragón, Aguilar, Navarro, y Ruiz, 2014). La finalidad principal de la prueba es evaluar el conocimiento numérico temprano y detectar aquellos alumnos que pueden mostrar riesgo de presentar Dificultades de Aprendizaje de las Matemáticas (DAM). Este test cuenta con tres versiones paralelas (A, B y C), concretamente en el presente estudio se trabajó con la versión B para evaluar al total del alumnado participante. Cada versión cuenta con 45 ítems y la puntuación máxima es de 45 puntos (uno por cada ítem correcto). El tiempo de administración oscila entre los 30 y 45 minutos y el modo de aplicación es individual. Dicho test está compuesto a su vez por dos subtest, el primero de ellos centrado en la evaluación de cuatro tipos de conceptos relacionales como son: comparaciones, clasificaciones, correspondencia y seriaciones, cada uno ellos evaluado a través de 5 ítems. Por otro lado, el test dispone de un subtest que evalúa conceptos de 
tipo numérico como son: conteo verbal, conteo estructurado, conteo resultante (el niño tiene que contar conjuntos de objetos sin señalar), conocimiento general de los números (el evaluado debe sumar los puntos que ha sacado al lanzar dos dados y colocar la ficha en el tablero del juego de la oca según corresponda) y estimación de números en una recta numérica, cada uno de ellos evaluado mediante 5 ítems. El alfa de Cronbach de esta prueba fue .90.

Prueba de fluidez de cálculo (Canals, Carbonell, Estaún, y Añaños, 1991). Con el fin de analizar la fluidez (definida como la velocidad y la precisión a la hora de efectuar las operaciones, Cowan y Powell, 2014), se seleccionó una prueba que tuviese en cuenta ambos aspectos a la hora de realizar el cálculo. Esta prueba incluye 64 operaciones, presentadas en disposición horizontal (p.e.: $5+2=$ ?). Los participantes deben resolver tantas operaciones como les sea posible en un tiempo máximo de un minuto. Esta tarea presenta cuatro ítems de entrenamiento, con el fin de que el evaluador constate que el alumno comprende adecuadamente la tarea. Una vez el alumno resuelve los ítems de prueba, el evaluador comienza a cronometrar el minuto de duración de la prueba. Cada operación correcta se puntúa con 1 y 0 la incorrecta. La puntuación total es el número de aciertos en el tiempo estipulado. Esta prueba está compuesta por subtests que evalúan la fluidez en las cuatro operaciones básicas. Para este trabajo se seleccionó la subprueba de sumas, atendiendo a la edad de los participantes.

\section{Procedimiento}

El presente estudio se realizó en dos años consecutivos, en base a dos sesiones de evaluación. Una primera sesión de evaluación se llevó a cabo a final del año escolar del último curso de Educación Infantil y la segunda sesión en la misma fecha (entre los meses de mayo y junio) mientras los alumnos cursaban primer curso se Educación Primaria. En la primera sesión los alumnos fueron evaluados mediante el TEMT-i para evaluar su competencia matemática temprana. En la segunda sesión, un año después, fue evaluada su fluidez de cálculo mediante la prueba de sumas de Canals. Ambas evaluaciones se llevaron a cabo por personal entrenado y especializado. Las distintas fases de la investigación se llevaron a cabo una vez obtenido el consentimiento informado de profesores y padres del alumnado.

\section{Análisis Estadístico}

El objetivo del presente estudió fue analizar la predicción de la fluidez de cálculo en Educación Primaria. En consecuencia, se perseguía conocer el valor predictivo de los precur- 
sores específicos de los rendimientos matemáticos, tanto relacionales como numéricos, evaluados a partir del TEMT-i. Para ellos, se seleccionó una técnica de análisis multivariante de regresión lineal múltiple por pasos (stepwise), con el fin de establecer en qué medida ciertas variables consideradas por la literatura predictoras de la competencia matemática se relacionaban con la variable criterio, es decir, la fluidez en el cálculo.

\section{Resultados}

En primer lugar en relación al objetivo principal de la investigación que es aportar información sobre la predicción de la fluidez de cálculo en Eduación Infantil, se estudiaron los estadísticos descriptivos de las variables evaluadas (tabla 1).

Tabla 1. Estadísticos descriptivos de las variables predictoras y criterio.

\begin{tabular}{lll}
\hline & Media & D.T. \\
\hline Fluidez de cálculo & 7.57 & 2.67 \\
\hline Comparaciones & 4.66 & .63 \\
\hline Clasificaciones & 2.16 & 1.04 \\
\hline Correspondencia & 3.35 & 1.19 \\
\hline Seriaciones & 2.22 & 1.11 \\
\hline Conteo verbal & 2.75 & 1.01 \\
\hline Conteo estructurado & 3.13 & 1.27 \\
\hline Conteo resultante & 2.55 & 1.21 \\
\hline Conocimiento general de los números & 2.94 & 1.04 \\
\hline Estimación & 1.03 & 1.26 \\
\hline
\end{tabular}

De manera previa a la realización del análisis de regresión se comprobó que la muestra cumplía los supuestos y requisitos para el uso de dicho análisis estadístico.

Tabla 2. Modelo de regresión lineal múltiple por pasos.

\begin{tabular}{|c|c|c|c|c|c|c|c|c|}
\hline \multirow[b]{2}{*}{ Modelo } & \multirow[b]{2}{*}{$R$} & \multirow[b]{2}{*}{$R^{2}$} & \multirow[b]{2}{*}{$\begin{array}{l}R^{2} \\
\text { corregida }\end{array}$} & \multicolumn{4}{|c|}{ Estadísticos de cambio } & \multirow[b]{2}{*}{$\begin{array}{l}\text { Durbin } \\
\text { Watson }\end{array}$} \\
\hline & & & & $\begin{array}{l}\text { Error típ } \\
\text { estimación }\end{array}$ & $\begin{array}{l}\text { Cambio } \\
\text { en } R 2\end{array}$ & $\begin{array}{l}\text { Cambio } \\
\text { en } F\end{array}$ & $\begin{array}{l}\text { Sig. } \\
\text { Cambio } \\
F\end{array}$ & \\
\hline 1 & $.494 a$ & .244 & .237 & 2.33 & .244 & 38.65 & .000 & \\
\hline 2 & $.521 b$ & .272 & .259 & 2.29 & .028 & 4.57 & .000 & 1.895 \\
\hline 3 & $.544 \mathrm{c}$ & .296 & .279 & 2.26 & .025 & 4.16 & .000 & \\
\hline
\end{tabular}


Nota: a. Variables predictoras: (Constante), Conteo Verbal; b. Variables predictoras: (Constante), Conteo Verbal, Conteo Resultante; c. Variables predictoras: (Constante), Conteo Verbal, Conteo Resultante, Estimación; d. Variable dependiente: Fluidez de cálculo.

Como se puede observar en la Tabla 2, derivados del análisis multivariante de regresión múltiple por pasos, surgieron tres modelos, cada uno con su propia capacidad explicativa. De ellos, fue el tercer modelo el que ofrecía una mayor capacidad explicativa. En consecuencia atendiendo a los valores de $\mathrm{R}^{2}$ el $29.6 \%$ de la varianza en el resultado de la fluidez en el cálculo pudo ser explicada por las tres variables introducidas en el último modelo, es decir, conteo verbal, conteo resultante y estimación. No obstante, el valor de $\mathrm{R}^{2}$ corregido, es decir, teniendo en cuenta el número de variables y sujetos implicados en el estudio mostró que el $27.9 \%$ de la varianza en la fluidez de cálculo podía ser predicha por las tres variables introducidas en el modelo de regresión.

Tabla 3. Coeficientes del modelo de regresión lineal múltiple.

\begin{tabular}{|c|c|c|c|c|c|c|c|}
\hline \multirow[b]{2}{*}{ Modelo } & \multicolumn{2}{|c|}{$\begin{array}{l}\text { Coeficientes no } \\
\text { estandarizados }\end{array}$} & \multirow{2}{*}{$\begin{array}{l}\text { Coeficientes } \\
\text { tipificados } \\
\text { Beta }\end{array}$} & \multirow[b]{2}{*}{$t$} & \multirow[b]{2}{*}{ Sig. } & \multicolumn{2}{|c|}{$\begin{array}{l}\text { Estadísticos de } \\
\text { colinealidad }\end{array}$} \\
\hline & $B$ & Error típ. & & & & $\begin{array}{l}\text { Toleran- } \\
\text { cia }\end{array}$ & $F I V$ \\
\hline 3 (Constante) & 3.418 & .629 & & 5.436 & .000 & & \\
\hline Conteo Verbal & .998 & .239 & .377 & 4.175 & .000 & .731 & 1.368 \\
\hline Conteo Resultante & .419 & .198 & .191 & 2.119 & .036 & .736 & 1.358 \\
\hline Estimación & .336 & .165 & .159 & 2.040 & .044 & .986 & 1.014 \\
\hline
\end{tabular}

En la Tabla 3 se recogen los distintos coeficientes del modelo de regresión. Podemos observar cómo el valor de $t$ se asociaba a una probabilidad de error inferior a .05 en las tres variables incluidas en el modelo predictivo. Asimismo, los resultados de la prueba $t$ y sus valores críticos contribuyeron a contrastar la hipótesis nula de que el coeficiente de regresión arrojaba un valor cero. Ante los resultados obtenidos, se asumió que las tres variables que formaban parte del modelo favorecían la explicación de la varianza de la variable dependiente. Del mismo modo, los coeficientes estandarizados arrojaron información acerca de cuáles de las variables introducidas en el modelo presentaban un mayor peso en la predicción de la variable dependiente, es decir, la fluidez de cálculo. Según los coeficientes beta recogidos en la Tabla 3, el conteo verbal $(\beta=.377)$, resultante $(\beta=.191)$ y estimación $(\beta=.159)$, fueron la 
variables que explicaron en mayor medida la fluidez de cálculo al año siguiente; es decir, en primer curso de Educación Primaria. Estos coeficientes señalaron al conteo verbal como el predictor de mayor peso de la fluidez en el cálculo, seguido del conteo resultante y la estimación, aunque éstas tuvieron un menor peso predictivo.

Es importante mencionar que del subtest numérico las variables conteo estructurado y conocimiento general de los números, se excluyeron del modelo, al igual que el total de las variables de tipo relacionales: comparaciones, clasificaciones, correspondencia y seriación, evaluadas a partir del TEMT-i como se muestra en la Tabla 4.

Tabla 4. Coeficientes estandarizados y valores de la prueba $t$ y significación estadística para las variables excluidas del modelo.

\begin{tabular}{llll}
\hline & $B$ & $t$ & Sig. \\
\hline Subtest Relacional & & & \\
\hline Comparaciones & -.064 & -.801 & .425 \\
\hline Clasificaciones & -.124 & -1.575 & .118 \\
\hline Correspondencia & .018 & .216 & .829 \\
\hline Seriaciones & .065 & .015 & .988 \\
\hline Subtest Numérico & & & \\
\hline Conteo Estructurado & .001 & .015 & .988 \\
\hline Conocimiento General de los Números & .024 & .278 & .782 \\
\hline
\end{tabular}

A la hora de establecer un modelo estadístico es importante comprobar su validez, por se estudió la independencia de los residuos a través del estadístico $D$ de Durbin-Watson, que presentó un valor $D=1.895$, confirmando con este valor cercano a 2 la ausencia de autocorrelación positiva (valores próximos a 0) y negativa (valores próximos a 4). Del mismo modo, se asumió también la ausencia de colinealidad y, por tanto, la estabilidad de las estimaciones al obtener altos valores de tolerancia y bajos de FIVs recogidos en la Tabla 3.

\section{Discusión y Conclusiones}

Los resultados obtenidos apoyan el hecho de que las habilidades matemáticas tempranas evaluadas al final del ciclo de Educación Infantil tienen un impacto considerable en la predic- 
ción de la fluidez de cálculo en el primer curso de Educación Primaria. Las tres variables que mayor peso presentaron en la predicción de la variable dependiente correspondieron al subtest numérico. Los resultados fueron los esperados debido a que según los postulados de Piaget (Piaget y Szeminska, 1943) las habilidades lógicas, como por ejemplo, clasificación o seriación, son la base fundamental para adquirir el concepto de número. Sin embargo, conforme se alcanzan aprendizajes más sofisticados, van perdiendo importancia en la explicación de los mismos.

En nuestra investigación de los dos tipos de habilidades matemáticas estudiadas, y que constituyen la base de la competencia numérica temprana, sólo las estrictamente numéricas predijeron la fluidez de cálculo. Concretamente, el conteo verbal, el conteo resultante y las estimación arrojaron en este orden un mayor peso y fueron introducidas en el modelo predictivo. Estos resultados pueden deberse a que el conteo verbal, entendido como la habilidad para recitar la secuencia numérica adecuadamente, es un requisito esencial en el desarrollo de estrategias de cálculo (Johansson, 2005). En consecuencia, el conteo fluido y preciso parece crítico para formar asociaciones en la memoria a largo plazo entre el problema presentado y la respuesta. De este modo, es posible progresar desde el empleo de estrategias de cálculo como medio para resolver el problema, a un proceso mucho más eficiente como es la recuperación de la solución de la memoria.

Específicamente, el conteo verbal constituye un procedimiento de solución temprana de conflictos de carácter matemático, y conforme esta habilidad se va perfeccionando el estudiante llega a detectar regularidades en la secuencia numérica, favoreciendo el desarrollo de nuevas y más precisas estrategias para resolver problemas matemáticos (Koponen et al., 2007) y justificando el papel que desempeña en la presente investigación.

Noël y Rousselle (2011) propusieron que el conteo es la clave del éxito en matemáticas. Tanto es así, que las habilidades de conteo evaluadas en educación infantil llegan a predecir las habilidades matemáticas en primaria (vanMarle, Chu, Li, y Geary, 2014), al igual que se halló en nuestro trabajo. En numerosas ocasiones se han vinculado las habilidades de conteo temprano y un óptimo rendimiento en matemáticas (Locuniak et al., 2008). Por tanto, presentar habilidades de conteo insuficientes o rudimentarias se relaciona con dificultades en las 
matemáticas (Gersten et al., 2005) por ello, es conveniente trabajar estas destrezas de manera temprana con el fin de prevenir problemas futuros.

Por otro lado, la variable que predijo la fluidez de cálculo en segundo lugar fue el conteo resultante (o sin señalar). Dentro de este grupo de actividades de conteo se englobaban las tareas relacionadas con la subitización (tres tareas). Este constructo se refiere a la habilidad para determinar a golpe de vista cuántos elementos hay cuando se presenta una pequeña cantidad de objetos sin necesidad de contarlos (Baroody, 2011). En nuestro trabajo, así como en otras investigaciones, esta habilidad contribuyó a explicar las diferencias individuales en matemáticas (Geary et al., 2008), incluso al final de la escuela primaria (Reigosa-Crespo, González-Alemañy, León, Torres, Mosquera, et al., 2013). Ya que se puede entender la subitización, al igual que el conteo, como procesos destinados a la enumeración de cantidades exactas, se deben diferenciar atendiendo especialmente a los requerimientos de cada uno, mostrándose el conteo como un proceso mucho más lento y costoso que la subitización.

Uno de los trabajos más recientes que apoya el papel predictor de la subitización fue el elaborado por Reigosa-Crespo et al., (2013). En dicho trabajo, tanto el conteo como la subitización predijeron la fluidez de cálculo, aunque únicamente fue significativo el valor de la subitización. Por lo tanto, estas habilidades numéricas básicas se consideran predictores de dominio específico no únicamente como herramientas promotoras de la adquisición de la competencia matemática, sino también durante la etapa de educación primaria, modulando el aprendizaje de estas destrezas.

En último lugar, el tercer predictor incluido en modelo de regresión lineal fue la estimación. Al igual que en nuestro trabajo, Both y Siegler (2008) encuentran una importante relación explicativa entre la estimación numérica evaluada en primer curso y el rendimiento de los alumnos en artimética. De manera previa a la intervención, llevaron a cabo un estudio pretest sobre la interrelaciones entre ambas variables, la correlación fue alta entre estimación y la prueba de adición, empleándose una medida de sumas simples similar a la empleada en el presente trabajo. La correlación entre estimación y adiciones en el trabajo de Both et al., (2008) fue $(r=.41 ; p<.01)$ mucho más elevada que la obtenida entre la prueba de cálculo y el predictor de dominio general memoria a corto plazo $(r=.21 ; p<.05)$. Este hecho avala la importancia de los predictores de dominio específico sobre los de dominio general (Fuch, 
Geary et al., 2010) en la explicación del rendimiento matemático, y la necesidad de prestar atención a este tipo de factores en su predicción.

Del mismo modo, existen numerosas investigaciones a favor de la importancia de la estimación, por ejemplo, un estudio previo llevado a cabo por los mismos autores (Booth y Siegler, 2006) mostró que las diferencias individuales en el aprendizaje de la línea numérica correlacionó con el rendimiento en matemáticas en todos los cursos en los que se evaluó. Este hecho se debe a que la adquisición del conteo y la asimilación de las propiedades de la secuencia numérica, contribuyen a establecer las bases para el aprendizaje de los números de mayor magnitud, favoreciendo la construcción de la representación de los mismos en una línea numérica mental (Feigenson, Dehaene, y Spelke, 2004) y señalando, a su vez, que los tres factores definidos por esta investigación como predictivos de la fluidez de cálculo se encuentran íntimamente relacionados.

Como limitaciones del trabajo sería conveniente aportar información de alguna medida de control relacionada con los factores de dominio general, tales como la inteligencia o las funciones ejecutivas. Del mismo modo, se constata la ausencia de una evaluación de la fluidez de cálculo en edades posteriores a los 6 años. También se considera necesario evaluar operaciones más complejas, tales como multiplicaciones y divisiones, conforme avance la edad del alumnado. Ambas limitaciones constituyen un punto de partida para futuros estudios de investigación en esta línea de trabajo.

\section{Referencias}

Aragón, E. L., Delgado, I., Aguilar, M., Araújo, A. y Navarro, J. I. (2013). Estudio de la influencia de la inteligencia y el género en la evaluación matemática temprana. European Journal of Education and Psychology, 6(1), 5-18.

Aragón, E., Aguilar, M., Navarro, J. y Araújo, A. (2015). Efectos de la aplicación de un programa de entrenamiento específico para el aprendizaje matemático temprano en educación infantil. Revista Española de Pedagogía, 260, 99-113.

Aragón, E. L., Navarro, J.I., Aguilar, M. y Cerda, G. (2015). Predictores cognitivos del conocimiento numérico temprano en alumnado de 5 años. Revista de Psicodidáctica, 20(1), 83-97. doi: 10.1387/RevPsicodidact.11088

Araújo, A., Aragón, E., Aguilar, M., Navarro, J. y Ruiz, G. (2014). Un estudio exploratorio para la adaptación de la versión española revisada del "Early Numeracy Test-R" para 
evaluar el aprendizaje matemático temprano. European Journal of Education and Psychology, 7(2), 83-93.

Baroody, A. J., Bajwa, N. P. y Eiland, M. (2009). Why can’t Johnny remember the basic facts?. Developmental Disabilities Research Review, 15, 69-79. doi:10.1002/ddrr.45

Baroody, A.J. (2011). Learning: A framework. In F. Fennell (Ed.), Achieving fluency in special education and mathematics (pp. 15-58). Reston, VA: National Council of Teachers of Mathematics.

Booth, J. L. y Siegler, R. S. (2008). Numerical magnitude representations influence arithmetic learning. Child Development, 79(4), 1016-1031. doi:10.1111/j.14678624.2008.01173.x

Booth, J. L. y Siegler, R. S. (2006). Developmental and individual differences in pure numerical estimation. Developmental Psychology, 41, 189-201. doi:10.1037/00121649.41.6.189

Bull, R., Espy, K. A. y Wiebe, S. A. (2008). Short-term memory, working memory, and executive functioning in preschoolers: Longitudinal predictors of mathematical achievement at age 7 years. Developmental Neuropsychology, 33, 205-228. doi:10.1080/87565640801982312

Canals, R., Carbonell, F., Estaún, S. y Añaños, E. (1991). Proves psicopedagògiques d'aprenentatges instrumentals: Cicles Inicial i Mitjà. Barcelona: Editorial Onda.

Clements, D. H. y Sarama, J. (2009). Learning and teaching early math: The learning trajectories approach. New York: Routledge.

Clements, D. H. y Sarama, J. (2011). Early childhood mathematics intervention. Science, 333, 968-970. doi:10.1126/science.1204537

Cowan, R. y Powell, D. (2014). The contributions of domain-general and numerical factors to third-grade arithmetic skills and mathematical learning disability. Journal of Educational Psychology, 106 (1), 214-229. doi:10.1037/a0034097

De Smedt, B., Janssen, R., Bouwens, K., Verschaffel, L., Boets, B. y Ghesquiere, P. (2009). Working memory and individual differences in mathematics achievement: A longitudinal study from first grade to second grade. Journal of Experimental Child Psychology, 103, 186-201. doi:10.1016/j.jecp.2009.01.004

Feigenson, L., Dehaene S. y Spelke, E. (2004). Core systems of number. Trends in Cognitive Science, 8, 307-314. doi:10.1016/j.tics.2004.05.002 
Fuchs, L. S., Geary, D. C., Compton, D. L., Fuchs, D., Hamlett, C. L., Seethaler, P. M., ... Schatschneider, C. (2010). Do Different Types of School Mathematics Development Depend on Different Constellations of Numerical versus General Cognitive Abilities?. Developmental Psychology, 46(6), 1731-1746.

Fuchs, L. S., Fuchs, D., Compton, D.L., Powell, S. R., Seethaler, P. M., Capizzi, A. M., Schatschneider, C. y Fletcher, J. M. (2006). The cognitive correlates of third-grade skill in arithmetic, algorithmic computation, and arithmetic word problems. Journal of Educational Psychology. 98, 29-43. doi:10.1037/0022-0663.98.1.29

Geary, D. C., Hoard, M. K., Nugent, L. y Byrd-Craven, J. (2008). Development of number line representations in children with mathematical learning disability. Developmental Neuropsychology, 33, 277-299. doi:10.1080/87565640801982361

Gelman, R. y Gallistel, C. (1978). The Child's Understanding of Number. Cambridge, MA: Harvard University Press.

Gersten, R., Jordan, N. C. y Flojo, J. R. (2005). Early identification and interventions for students with mathematics difficulties. Journal of Learning Disabilities, 38, 293-304.

Ho, C. S. y Fuson, K. C. (1998). Children's knowledge of teen quantities as tens and ones: Comparisons of Chinese, British, and American Kindergartners. Journal of Educational Psychology, 90, 536-544. doi: http://dx.doi.org/10.1037/0022-0663.90.3.536

Johansson, B. S. (2005). Number-word sequence skill and arithmetic performance. Scandinavian Journal of Psychology, 46 (2), 157-167. doi:10.1111/j.14679450.2005.00445.x

Jordan, N., Kaplan, D., Locuniak, M. y Ramineni, C. (2007). Predicting first-grade math achievement from developmental number sense trajectories. Learning Disabilities Research y Practice, 22, 36-46. doi:10.1111/j.1540-5826.2007.00229.x

Kilpatrick, J., Swafford, J. y B. Findell (Eds.). (2001). Adding it up: Helping children learn mathematics. Washington DC: National Academies Press.

Koponen, T., Aunola, K., Ahonen, T. y Nurmi, J.-E. (2007). Cognitive predictors of singledigit and procedural calculation skills and their covariation with reading skill. Journal of Experimental Child Psychology, 97, 220-241. doi:10.1016/j.jecp.2007.03.001

Landerl, K., Bevan, A. y Butterworth, B. (2004). Developmental dyscalculia and basic numerical capacities: A study of 8-9-year-old students. Cognition, 93(2), 99-125. doi:10.1016/j.cognition.2003.11.004 
Locuniak, M. N. y Jordan, N. C. (2008). Using kindergarten number sense to predict calculation fluency in second grade. Journal of Learning Disabilities, 41, 451-459. doi:10.1177/0022219408321126

Martins-Mourao, A. y Cowan, R. (1998). The emergence of additive composition of number. Educational Psychology, 18, 377-390.

Mazzocco, M. M. M. y Thompson, R. E. (2005). Kindergarten predictors of math learning disability. Learning Disabilities Research y Practice, 20, 142-155. doi:10.1111/j.1540-5826.2005.00129.x

National Mathematics Advisory Panel. (2008). Foundations for success: The final report of the National Mathematics Advisory Panel. Washington, DC: U.S. Department of Education.

Navarro, J. I., Aguilar, M., García, M., Menacho, I., Marchena, E. y Alcalde, C. (2010). Diferencias en habilidades matemáticas tempranas en niños y niñas de 4 a 8 años. Revista Española de Pedagogía, 245, 85-98.

Noël, M.-P. y Rousselle, L. (2011). Developmental changes in the profiles of dyscalculia: An explanation based on a double exact-and-approximate number representation model. Frontiers in Human Neuroscience, 5, 1-4. doi:10.3389/fnhum.2011.00165

Núñez del Río, M. y Lozano Guerra, I. (2003). Evaluación del pensamiento matemático temprano en alumnos con déficit intelectual, mediante la prueba TEMA-2. Revista Española de Pedagogía, 226, 547-564.

Passolunghi, M. C., Lanfranchi, S., Altoè, G. y Sollazzo, N. (2015). Early numerical abilities and cognitive skills in kindergarten children. Journal of Experimental Child Psychology, 135, 25-42. doi:10.1016/j.jecp.2015.02.001

Piaget, J. y Szeminska, A. (1943). Génesis del Número en el niño. Buenos Aires: Guadalupe. Reigosa-Crespo, V., González-Alemañy, E., León, T., Torres, R., Mosquera, R. y ValdésSosa, M. (2013). Numerical capacities as domain-specific predictors beyond early mathematics learning: A longitudinal study. PLoS ONE, 8(11): e79711. doi:10.1371/journal.pone.0079711

Sarama, J. y Clements, D. H. (2009). Early childhood mathematics education research: Learning trajectories for young children. New York: Routledge.

Van Luit, J., Van De Rijt, B., Araújo, A., Aguilar, M., Aragón, E., Ruiz, G., ...GarcíaSedeño, M. (2015). Test de evaluación de la competencia matemática tempranarevisado (TEMT-i). Madrid: EOS. 
Van Marle, K., Chu, F. W., Li, Y. y Geary, D. C. (2014). Acuity of the approximate number system and preschoolers' quantitative development. Developmental Science, 17, 492505. doi:10.1111/desc. 12143 